\title{
PROPRIEDADES FÍSICAS DE UM ARGISSOLO APÓS FERTIRRIGAÇÃO COM VINHAÇA E SISTEMAS DE COLHEITA DA CANA DE AÇUCAR
}

Carlos Henrique dos Santos, Claudinei Alberto Cardin, José Eduardo Creste, Willian Lucas Mativi, Ana Carolina Mônico Moreira, Marcos Antonio Escarmínio

Universidade do Oeste Paulista - UNOESTE, Programa de Pós-Graduação em Agronomia, Presidente Prudente, SP. Email: chenrique@unoeste.br

\section{RESUMO}

O objetivo deste trabalho foi monitorar alterações no teor de matéria orgânica e nas propriedades físicas de um Argissolo após a fertirrigação com vinhaça e diferentes sistemas de colheita da cana de açúcar, no oeste paulista. A coleta de dados ocorreu em uma empresa de bioenergia de Presidente Prudente, SP, seguindo o delineamento inteiramente casualizado, em esquema fatorial $5 \times 5$, caracterizado pelos manejos sem aplicação de vinhaça e colheita de cana sem queima; com aplicação de vinhaça e colheita de cana sem queima; com aplicação de vinhaça e colheita de cana queimada; sem aplicação de vinhaça e colheita de cana queimada; mata nativa, e pelas profundidades de amostragem de solo $(0-10,10-20,20-30,30-40,40-$ $50 \mathrm{~cm}$ ), em quatro repetições. A área mata nativa (MN) foi utilizada como referência para demonstração das alterações nas propriedades químicas e físicas do solo pela aplicação dos manejos. Nas amostras coletadas foram determinados os parâmetros matéria orgânica, densidade do solo, densidade de partículas, porosidade total e umidade de saturação. Os dados foram submetidos à análise de variância e as médias foram comparadas pelo teste de Tukey, ao nível de $5 \%$ de probabilidade. $O$ teor de matéria orgânica e a densidade do solo não foram influenciados pela aplicação de vinhaça, formas de colheita de cana crua ou queimada. A densidade do solo foi significativamente menor na camada de 0-10 cm, independente do manejo. A porosidade total e a umidade de saturação do solo foram reduzidas a partir de $20 \mathrm{~cm}$ de profundidade, independente do manejo. A fertilidade e a física de solo devem ser monitoradas por um período maior tempo.

Palavras-chave: fertilidade de solo; matéria orgânica; compactação; retenção de água; solo arenoso.

\section{PHYSICAL PROPERTIES OF AN ARGISOL AFTER FERTIRRIGATION WITH VINASSE AND HARVEST SYSTEM OF SUGAR CANE}

\begin{abstract}
The aim of this work was to evaluate the changes in the organic matter content and physical properties of an Argisol after fertigation with vinasse and the different hasvesting systems of sugar cane, in the west of Sao Paulo state. Data collection was carried out in a bioenergy company in Presidente Prudente, SP, following the completely randomized design, in a $5 \times 5$ factorial scheme, characterized by the management without vinasse application and harvest of sugar cane without burning; with vinasse application and harvest of sugar cane without burning; with vinasse application and harvest of sugar cane burned; without vinasse application and harvest of sugar cane burned; plus native forest and soil sampling depths $(0-1010-20,20-$ $30,30-40,40-50 \mathrm{~cm}$ ), with four replications. The MN area was used as a control to demonstrate the changes in the chemical and physical properties of the soil by applying the treatments. The organic matter soil density, particle density, total porosity and saturation moisture were determined in the collected samples. Data were submitted to analysis of variance and the means were compared by the Tukey test, at a $5 \%$ probability level. The organic matter content and soil density were not influenced by the managements studied. Soil density was significantly lower in the 0-10 cm layer, regardless of the management. The total porosity and soil saturation moisture were reduced from $20 \mathrm{~cm}$ depth, regardless of the management. The change in soil use from the native to the agricultural area condition negatively influenced all evaluated parameters. The evaluation of fertility and soil physics should be considered in long-term studies.
\end{abstract}

Keywords: soil fertility; organic matter; soil compaction; water retention; sandy soil. 


\section{INTRODUÇÃO}

A cana de açúcar é uma cultura de fundamental importância para o agronegócio e para o setor bioenergético brasileiro. A área total cultivada na safra 2017/2018 foi estimada em 8,8 milhões de hectares, com produção próxima de 636 milhões de toneladas de colmos (CONAB, 2017). Dentro deste contexto, destaca-se que a região Sudeste foi responsável pelo processamento de 415 milhões de toneladas de colmos e, o estado de São Paulo responsável por $84 \%$ deste total.

A cultura também assumiu posição de destaque para o agronegócio paulista, uma vez que houve ampliação significativa das áreas de cultivo no decorrer dos anos (CONAB, 2017) em detrimento da redução das áreas de outras monoculturas, além da cobertura nativa (OLIVEIRA et al., 1995). Salienta-se, porém, que o avanço do cultivo da cana de açúcar proporciona condições para que as práticas contínuas de manejo intensivo modifiquem as propriedades químicas, físicas e biológicas do solo (ABIVEN et al., 2009; SOUZA et al., 2005), podendo iniciar um processo de degradação do mesmo (BRONICK; LAL, 2005). Por isso, o monitoramento da qualidade física dos solos têm se tornado constante (LIMA et al., 2013).

O manejo tradicional da cultura promove alterações como o aumento da densidade e a diminuição da porosidade e da taxa de infiltração de água no solo quando comparados com o ambiente sob mata nativa (VASCONCELOS et al., 2014). Mas, benefícios também foram observados, pois resultados de pesquisa indicaram que a aplicação de vinhaça pode ter menor impacto na degradação física do solo em virtude da adição de matéria orgânica (OLIVEIRA et al., 2010).

A colheita da cana de açúcar após a queimada também propicia a perda da qualidade física do solo, diante da redução do teor de matéria orgânica e, consequentemente, de sua agregação (MARQUES et al., 2004). Em função deste e de outros fatores, a eliminação total da queima dos canaviais paulistas está prevista para 2031, nas áreas com declividade superior a $12 \%$ e ou menores de 150 ha, e para 2021 nas colhidas mecanicamente. Enquanto a prática da queima não é eliminada, na safra 2016/2017, 90\% da área cultivada no estado de São Paulo foi colhida mecanicamente sem queima (IEA, 2017), colaborando para o incremento de palhada na superfície do solo e na melhoria das suas características físicas. A qualidade do solo está embasada na interação das suas propriedades químicas, físicas e biológicas permitindo ao mesmo desempenhar melhor suas funções dentro do sistema solo-planta-atmosfera (VEZZANI; MIELNICZUK, 2009).

Com base nestas informações, este estudo objetivou conhecer a ação da aplicação da vinhaça e das formas de colheita manual e mecanizada após a queima e sem a queima da cana de açúcar nos parâmetros físicos e no teor de matéria orgânica do solo, no oeste paulista.

\section{MATERIAL E MÉTODOS}

Os dados desta pesquisa foram coletados nas áreas de produção agrícola, na safra 2012/2013, de uma empresa do setor de bioenergia, no distrito de Ameliópolis, em Presidente Prudente/SP, nas coordenadas geográficas de $21^{\circ} 51^{\prime} 57^{\prime \prime} S$ (latitude), e $51^{\circ} 15^{\prime} 35^{\prime \prime} \mathrm{W}$ (longitude) e 329,4 m de altitude. $\mathrm{O}$ clima regional é Aw, segundo a caracterização internacional de Köppen, com precipitação pluviométrica e temperatura média anual de $1.858,7 \mathrm{~mm}$ e $25,5^{\circ} \mathrm{C}$ em 2012 , e $1.633,6 \mathrm{~mm}$ e $24,8^{\circ} \mathrm{C}$ em 2013 , respectivamente.

As coletas de terra foram realizadas em áreas de manejo com a cultivar RB867515, no terceiro corte, e em solo classificado como Argissolo Vermelho-Amarelo com teores médios de 840,55 e $105 \mathrm{~g} \mathrm{~kg}^{-1}$ de areia total, silte e argila, respectivamente, na profundidade de $0-25$ $\mathrm{cm}$, e de 793, 62 e $145 \mathrm{~g} \mathrm{~kg}^{-1}$ de areia total, silte e argila, respectivamente, na profundidade de 25 $50 \mathrm{~cm}$ (EMBRAPA, 1997).

A amostragem de solo seguiu o delineamento estatístico de parcelas subdivididas, em esquema fatorial $5 \times 5$, com quatro repetições, sendo os manejos considerados como as parcelas e as profundidades as subparcelas. Os quatro manejos adotados na cana de açúcar, além da mata nativa, e as cinco profundidades de solo amostradas constituíram o primeiro e o segundo fatores, respectivamente. O primeiro fator foi descrito como: (A) SVSQ: Área de cana de açúcar sem aplicação de vinhaça (SV) e colheita mecanizada sem queima (SQ); (B) CVSQ: Cana de açúcar com aplicação de vinhaça (CV) e colheita mecanizada sem queima (SQ); (C) CVCQ: Cana de açúcar com aplicação de vinhaça (CV) e colheita manual queimada (CQ); (D) SVCQ: Cana de açúcar sem aplicação de vinhaça (SV) e colheita manual queimada (CQ); (E) Área de mata nativa 
remanescente (MN) utilizada como parâmetro das alterações nas propriedades químicas e físicas do solo diante dos manejos adotados na cultura. As áreas A, B, C e D apresentaram, respectivamente, 8,$7 ; 8,4 ; 4,2 ; 5,2$ ha e cultivo de cana-de-açúcar nos últimos 20 anos. A MN remanescente foi dimensionada em 10,2 ha. 0 segundo fator representa as profundidades de 0 $10,10-20,20-30,30-40$ e $40-50 \mathrm{~cm}$.
Anteriormente ao plantio nestas áreas, a empresa procedeu à amostragem de solo nas profundidades de $0-25$ e $25-50 \mathrm{~cm}$, seguindo os métodos descritos por Raij et al. (2001), para a caracterização química e estabelecimento dos critérios de correção e adubação do solo (Tabelas 1 e 2).

Tabela 1. Análise química de solo $(0-25 \mathrm{~cm})$ das áreas de manejo coletadas antes do plantio da cana de açúcar, 2009

\begin{tabular}{cccccccccccc}
\hline \multirow{2}{*}{ Área } & $\mathrm{pH}$ & $\mathrm{MO}$ & $\mathrm{P}_{\text {resina }}$ & $\mathrm{K}$ & $\mathrm{Ca}$ & $\mathrm{Mg}$ & $\mathrm{H}+\mathrm{Al}$ & $\mathrm{Al}$ & $\mathrm{SB}$ & $\mathrm{CTC}$ & $\mathrm{V}$ \\
\cline { 2 - 12 } & $\left(\mathrm{CaCl}_{2}\right)$ & $\mathrm{g} \mathrm{dm}^{-3}$ & $\mathrm{mg} \mathrm{dm}^{-3}$ & \multicolumn{7}{c}{$-0-25 \mathrm{~cm}$} \\
\hline SVSQ & 4,4 & 13 & 5 & 1,3 & 11 & 3 & 18 & 2 & 15 & 33 & 46 \\
SVCQ & 4,1 & 11 & 9 & 1,0 & 10 & 2 & 20 & 3 & 13 & 37 & 40 \\
CVSQ & 4,8 & 15 & 10 & 3,7 & 12 & 4 & 16 & 1 & 20 & 36 & 55 \\
CVCQ & 4,8 & 15 & 10 & 3,7 & 12 & 4 & 16 & 1 & 20 & 36 & 55 \\
MN & 4,8 & 12 & 23 & 2,8 & 12 & 4 & 15 & 1 & 19 & 34 & 56 \\
\hline
\end{tabular}

Tabela 2. Análise química de solo $(25-50 \mathrm{~cm})$ das áreas de manejo coletadas antes do plantio da cana de açúcar, 2009

\begin{tabular}{|c|c|c|c|c|c|c|c|c|c|c|c|}
\hline \multirow{2}{*}{ Área } & $\mathrm{pH}$ & MO & $P_{\text {resina }}$ & $\mathrm{K}$ & $\mathrm{Ca}$ & $\mathrm{Mg}$ & $\mathrm{H}+\mathrm{Al}$ & Al & SB & CTC & V \\
\hline & $\left(\mathrm{CaCl}_{2}\right)$ & $\mathrm{g} \mathrm{dm}^{-3}$ & $\mathrm{mg} \mathrm{dm^{-3 }}$ & \multicolumn{7}{|c|}{---------------------- $\mathrm{mmol}_{\mathrm{c}} \mathrm{dm}^{-3}$} & $\%$ \\
\hline \multicolumn{12}{|c|}{$25-50 \mathrm{~cm}$} \\
\hline SVSQ & 4,5 & 13 & 7 & 1,3 & 13 & 3 & 17 & 2 & 17 & 34 & 49 \\
\hline SVCQ & 4,0 & 10 & 9 & 0,8 & 12 & 4 & 25 & 4 & 17 & 42 & 40 \\
\hline CVSQ & 4,5 & 10 & 22 & 1,5 & 14 & 6 & 17 & 3 & 19 & 37 & 53 \\
\hline CVCQ & 4,5 & 10 & 22 & 1,5 & 14 & 6 & 17 & 3 & 22 & 39 & 55 \\
\hline $\mathrm{MN}$ & 4,8 & 12 & 23 & 2,8 & 12 & 4 & 15 & 1 & 19 & 34 & 56 \\
\hline
\end{tabular}

Posteriormente, a cada ano, análises de solo nas mesmas profundidades foram efetuadas para realização da adubação e correção do solo, visando o melhor desenvolvimento das soqueiras. As áreas que não receberam aplicação de vinhaça foram adubadas com $400 \mathrm{~kg} \mathrm{ha}^{-1}$ do formulado comercial 05-26-30, o que disponibilizou $120 \mathrm{~kg}$ $\mathrm{ha}^{-1}$ de $\mathrm{K}_{2} \mathrm{O}$. Todas as áreas manejadas com vinhaça receberam, aproximadamente, 61,0 (2010); 71,1 (2011); 92,5 (2012) e $83,3 \mathrm{~m}^{3} \mathrm{ha}^{-1}$ (2013), de acordo com o teor de K encontrado no solo.

A amostragem para avaliação dos parâmetros químicos e físicos do solo foi realizada em março de 2013, após o terceiro corte da cana de açúcar, nas áreas previamente definidas e georreferenciadas.

A determinação do teor de matéria orgânica (MOS) foi realizada em amostras deformadas de solo, coletadas nas profundidades de 0-10, 10-20, 20-30, 30-40 e 40-50 cm, em quatro trincheiras, abertas nas dimensões de 50 (C) $\times 30(\mathrm{~L}) \times 80 \mathrm{~cm}(\mathrm{P})$ e distanciadas $50 \mathrm{~m}$ entre as mesmas. Duas amostras simples, uma de cada lado da trincheira, foram coletadas em todas as profundidades e misturadas para obtenção de uma amostra composta representativa, que foram submetidas à análise química para a extração da MOS pelo método de Walkley-Black e quantificação pelo método colorimétrico (RAIJ et al., 2001), em espectrofotômetro FEMTO, modelo 600 Plus. Estas amostras deformadas também foram utilizadas para a determinação da densidade de partículas (Dp), $\mathrm{g} \mathrm{cm}^{-3}$, calculada a partir da equação 1: 


$$
D p=\frac{M s}{(V a-V b)} \quad(\text { Equação } 1)
$$

onde: Ms = Massa de solo deformado e seco (g); $\mathrm{Va}=$ Volume do erlenmeyer de $50 \mathrm{~mL}$ calibrado com balão volumétrico de $50 \mathrm{~mL}$; $\mathrm{Vb}=$ Volume de álcool etílico gasto (mL) (EMBRAPA, 1997).

Os valores de densidade do solo (Ds) foram obtidos a partir da coletada de duas amostras indeformadas em cada profundidade, sendo uma de cada lado das trincheiras, utilizando-se cilindros de aço inox com volume de $92 \mathrm{~cm}^{3}$. 0 cálculo da $D_{\mathrm{s}}\left(\mathrm{g} \mathrm{cm}^{-3}\right)$ foi realizado utilizando a equação 2 :

$$
D s=\frac{m}{v} \quad(\text { Equação 2) }
$$

onde $\mathrm{m}=$ massa de solo seco $(\mathrm{g}) ; \mathrm{v}=$ volume do anel de aço inox $\left(\mathrm{cm}^{3}\right)$ (EMBRAPA, 1997).

As amostras indeformadas de solo também foram utilizadas para a determinação da umidade de saturação. Para isso, os conjuntos de solo e anel foram pesados, em duplicata, obtendo-se a massa natural. Posteriormente, a amostra foi colocada em bandeja contendo água até $2 / 3$ da sua altura, durante 24 horas, para a saturação completa. Um fragmento de tecido de poliéster com malha de 120 fios $\mathrm{cm}^{-2}$ foi fixado com elástico na parte inferior do anel para evitar a perda de solo. O cálculo da umidade de saturação $(\theta \mathrm{s})$, em $\mathrm{cm}^{3} \mathrm{~cm}^{-3}$, foi realizado utilizando a equação 3 :

$$
\theta s=100 *\left\{\frac{(M a-M b)}{V}\right\} \quad(\text { Equação 3) }
$$

onde: $\mathrm{Ma}=$ massa do solo saturado $(\mathrm{g}) ; \mathrm{Mb}=$ massa de solo seco $105{ }^{\circ} \mathrm{C}(\mathrm{g}) ; \mathrm{V}=$ volume da amostra $\left(\mathrm{cm}^{3}\right)$ (EMBRAPA, 1997).

Com os resultados de Ds e de Dp foi possível calcular a porosidade total $(\mathrm{Pt})$ do solo, $\mathrm{em} \mathrm{cm}^{3} \mathrm{~cm}^{-3}$, utilizando a equação 4 :

$$
P t=1-\left(\frac{D s}{D p}\right) \quad(\text { Equação 4) }
$$

onde: $\mathrm{Ds}=$ Densidade do solo $\left(\mathrm{g} \mathrm{cm}^{-3}\right) ; \mathrm{Dp}=$ Densidade de partículas $\left(\mathrm{g} \mathrm{cm}^{-3}\right)$ (EMBRAPA, 1997).

Os resultados obtidos foram submetidos à análise de variância e as médias foram comparadas pelo teste de Tukey, a 5\% de probabilidade. A significância da interação entre os fatores de manejo e profundidades de solo foi verificada pelo teste $F$.

\section{RESULTADOS E DISCUSSÃO}

$A$ análise do teste $F$ revelou que os parâmetros físicos densidade do solo (Ds), densidade de partículas (Dp), porosidade total $(\mathrm{Pt})$ e umidade de saturação $(\theta \mathrm{s})$ foram influenciados significativamente pelos manejos nas profundidades avaliadas e, que também houve ação direta destes fatores, e de sua interação, no teor de matéria orgânica do solo (MOS) e na $\theta$ s (Tabela 3).

Tabela 3. Resultados do teste $F$, calculados para os parâmetros físicos e para a MOS nos sistemas de manejo e nas profundidades avaliados

\begin{tabular}{cccccc}
\hline FV & Ds & Dp & Pt & $\theta s$ & MOS \\
\hline Manejos & $55,36^{* *}$ & $3,10^{*}$ & $19,36^{* *}$ & $142,03^{* *}$ & $38,62^{*}$ \\
Profundidade & $9,99^{* *}$ & $0,44^{\text {ns }}$ & $3,46^{*}$ & $29,28^{* *}$ & $52,85^{* *}$ \\
Manejo x Profundidade & $0,83^{\text {ns }}$ & $*^{*} 0,72^{\text {ns }}$ & $1,00^{\text {ns }}$ & $2,55^{* *}$ & $14,04^{* *}$ \\
\hline
\end{tabular}

** significativo ao nível de $1 \%$ de probabilidade $(p<0,01) ; *$ significativo ao nível de $5 \%$ de probabilidade $(0,01=<p<$ $0,05)$; ns - não significativo ( $p>=0,05)$.

A aplicação de vinhaça e a forma de colheita da cana de açúcar não reduziram significativamente a Ds durante quatro anos de manejos, destacando, porém, que nas áreas agrícolas o valor foi estatisticamente superior ao apresentado pela MN (Tabela 4). Estes resultados são corroborados por Viana et al. (2011), os quais destacaram que a Ds está associada ao tráfego de máquinas excessivo e à diminuição do teor de MOS no decorrer do tempo. A Ds é uma das propriedades que estão especificamente relacionadas à organização estrutural do solo, e as alterações nesta são melhor avaliadas diante da pressão exercida pelos sistemas de manejo (OLIVEIRA et al., 2014) em função do tempo (CEDDIA et al., 1999). Já o teor de MOS tem papel fundamental nesta estruturação (VASCONCELOS et al. 2014), pois a sua grande capacidade de retenção de água exercida reduz a quantidade de água retida pela matriz do solo, sendo necessária maior quantidade água para que a densidade máxima de compactação seja atingida (CAMARGO; ALLEONI, 1997). 
Tabela 4. Valores do teste de médias, calculados para os parâmetros físicos do solo nos sistemas de manejo e nas profundidades avaliados

\begin{tabular}{|c|c|c|c|c|}
\hline \multirow{2}{*}{ Manejos } & Ds & $\mathrm{Dp}$ & $\mathrm{Pt}$ & $\theta \mathrm{s}$ \\
\hline & \multicolumn{2}{|c|}{$\begin{array}{ll}\text { Ds } & \text { Dp } \\
-\cdots & \left(\mathrm{g} \mathrm{cm}^{-3}\right) \text {---- }\end{array}$} & \multicolumn{2}{|c|}{$---\left(\mathrm{cm}^{3} \mathrm{~cm}^{-3}\right)$} \\
\hline SV SQ & $1,72 \mathrm{a}$ & $2,36 \mathrm{~b}$ & $26,39 c$ & $0,28 d$ \\
\hline SV CQ & $1,67 a b$ & $2,52 \mathrm{a}$ & $33,40 \mathrm{~b}$ & $0,28 d$ \\
\hline CV SQ & $1,68 a b$ & $2,36 \mathrm{ab}$ & $28,59 \mathrm{bc}$ & $0,31 \mathrm{~b}$ \\
\hline CV CQ & $1,65 b$ & $2,46 a b$ & $32,65 b$ & $0,29 c$ \\
\hline $\mathrm{MN}$ & $1,38 c$ & $2,35 \mathrm{~b}$ & $41,06 \mathrm{a}$ & $0,39 \mathrm{a}$ \\
\hline \multicolumn{5}{|c|}{ Profundidade $(\mathrm{cm})$} \\
\hline $0-10$ & $1,52 b$ & $2,39 a$ & $36,32 \mathrm{a}$ & $0,35 \mathrm{a}$ \\
\hline $10-20$ & $1,62 \mathrm{a}$ & $2,41 a$ & 32,45 a & $0,31 \mathrm{~b}$ \\
\hline $20-30$ & $1,67 \mathrm{a}$ & $2,45 a$ & $31,52 a b$ & $0,30 \mathrm{~b}$ \\
\hline $30-40$ & $1,66 \mathrm{a}$ & $2,37 \mathrm{a}$ & $31,90 a b$ & $0,30 \mathrm{~b}$ \\
\hline $40-50$ & $1,64 \mathrm{a}$ & $2,42 a$ & $29,90 \mathrm{~b}$ & $0,31 \mathrm{~b}$ \\
\hline CV (\%) & 5,11 & 7,65 & 17,65 & 0,33 \\
\hline DMS & 0,07 & 0,16 & 5,06 & 0,015 \\
\hline
\end{tabular}

As médias seguidas pela mesma letra não diferem estatisticamente entre si. Foi aplicado o Teste de Tukey ao nível de $5 \%$ de probabilidade.

Pesquisas indicam que a aplicação de vinhaça tem aumentado o teor de MOS e minimizado os efeitos negativos nas propriedades físicas do solo (CANELLAS et al., 2003). Apesar desta afirmação, os resultados apresentados na Tabela 5 mostraram que não houve aumento significativo da MOS decorrente da aplicação de vinhaça. A sua rápida degradação em solos arenosos, principalmente, na presença deste resíduo, pode ser responsável por este efeito. A vinhaça adiciona água e nutrientes ao ambiente, aumentando a atividade microbiológica e, consequentemente, a taxa de decomposição dos resíduos orgânicos. De acordo com Andrioli (1986), o tempo de manejo assume mais importância do que a quantidade de vinhaça aplicada em pouco tempo, pois tal prática pode acelerar a degradação da palha. Segundo o pesquisador, a aplicação de $1200 \mathrm{~m}^{3} \mathrm{ha}^{-1}$ de vinhaça não foi capaz de modificar a Ds, justamente por não incrementar significativamente o teor de MOS. Zolin et al. (2011) confirmaram que a aplicação de vinhaça durante 20 anos proporcionou maiores teores de MOS mas, mesmo assim, não reduziu a Ds. Os fatos evidenciam que a conversão de áreas nativas em cultivadas influencia negativamente no balanço de MOS (BAYER et al., 2000; CARDIN et al., 2016), provocando reflexos negativos diretamente na estruturação do solo (BAYER et al., 2000).

Tabela 5. Teores de MOS, $\mathrm{g} \mathrm{dm}^{-3}$, considerando a interação entre os manejos e as profundidades de solo avaliados

\begin{tabular}{|c|c|c|c|c|c|c|c|c|c|c|}
\hline \multirow{3}{*}{$\begin{array}{c}\text { Manejos } \\
\text { SV SQ }\end{array}$} & \multicolumn{10}{|c|}{ Profundidade $(\mathrm{cm})$} \\
\hline & \multicolumn{2}{|c|}{$0-10$} & \multicolumn{2}{|c|}{$10-20$} & \multicolumn{2}{|c|}{$20-30$} & \multicolumn{2}{|c|}{$30-40$} & \multicolumn{2}{|c|}{$40-50$} \\
\hline & 9,55 & bA & 7,55 & $\mathrm{bA}$ & 6,95 & $\mathrm{aA}$ & 6,67 & $\mathrm{aA}$ & 6,60 & $a A$ \\
\hline SV CQ & 9,47 & bA & 8,70 & bA & 8,30 & $\mathrm{aA}$ & 6,93 & $\mathrm{aA}$ & 6,75 & $\mathrm{aA}$ \\
\hline CV SQ & 10,58 & bA & 9,15 & bAB & 7,45 & $a A B$ & 6,53 & $a B$ & 6,73 & $a B$ \\
\hline CV CQ & 11,83 & bA & 10,07 & $\mathrm{bAB}$ & 9,75 & $a A B$ & 7,20 & $a B$ & 6,55 & $a B$ \\
\hline $\mathrm{MN}$ & 29,00 & $a A$ & 16,50 & $a B$ & 9,15 & $\mathrm{aC}$ & 7,77 & $\mathrm{aC}$ & 6,85 & $\mathrm{aC}$ \\
\hline
\end{tabular}

$\mathrm{CV}(\%)$ geral = 20 (MOS); DMS para linhas (letras maiúsculas) e colunas (letras minúsculas) = 3,72 (MOS). Letras iguais, minúsculas na coluna e maiúsculas na linha, não diferem entre si a $5 \%$ de probabilidade pelo teste Tukey.

De forma geral, a Ds foi significativamente menor apenas na camada de 0-10 cm, independente do manejo (Tabela 4), embora não se tenha observado diferença significativa no teor de MOS (Tabela 5). Braida et al. (2006), Freddi et al. (2007) e Zolin et al. (2011) também verificaram alterações neste parâmetro na camada mais superficial do solo. Normalmente, 
os sistemas de manejo alteram a Ds somente até $10 \mathrm{~cm}$, destacando que no cultivo de cana com colheita mecanizada, o tráfego de máquinas, como as colhedoras e os transbordos, é o principal responsável por este efeito (GARBIATTE et al., 2011; SOUZA et al., 2012).

Independente da área de manejo, os valores de Ds não foram diferentes estatisticamente e variaram de 1,65 a $1,72 \mathrm{~g} \mathrm{~cm}^{-3}$ (Tabela 4). Garbiate et al. (2011) encontraram a mesma tendência de valores, sendo que nas áreas de colheita mecanizada com cana queimada a Ds atingiu $1,84 \mathrm{~g} \mathrm{~cm}^{-3}$. Compactações significativas, a partir de 1,50 até $1,60 \mathrm{~kg} \mathrm{dm}^{-3}$, na camada de $0-10 \mathrm{~cm}$ foram diagnosticadas, mesmo em solos arenosos, sob o manejo de colheita mecanizada sem queima comparado com a área queimada (LUCA et al., 2008). A mecanização agrícola, principalmente com as operações de preparo causa a desestruturação dos horizontes superficiais ou a compactação dos sub-superficiais. As operações de colheita são as que mais utilizam máquinas pesadas compactando o solo, principalmente quando utilizadas em condições inadequadas de umidade (OLIVEIRA et al., 2011). É importante frisar que, o monitoramento dos manejos de curta duração não indicam diferenças nos parâmetros físicos do solo a partir da mudança de sistema de colheita (OLIVEIRA et al., 2014), mas a adição constante de resíduos orgânicos ao solo é capaz de alterar seus atributos físicos com o decorrer do tempo (CANELLAS et al., 2003; OLIVEIRA et al., 2014).

A Dp, considerado um atributo físico estático do solo (OLIVEIRA et al., 2014), também não foi modificada diante da diferenciação dos manejos tampouco em função das profundidades avaliadas (Tabela 5). No entanto, Zolin et al.
(2011) destacaram tendência de diminuição da Dp, de 15 a $30 \mathrm{~cm}$, ao longo dos anos de aplicação de vinhaça, diante do aumento do teor de MOS nesta camada.

A Pt é um parâmetro que demonstra a correlação entre a Ds/Dp e, consequentemente a ação direta da compactação do ambiente. Houve diminuição significativa na $\mathrm{Pt}$ apenas entre os manejos agrícolas e a $\mathrm{MN}$, com redução dos valores a partir de $20 \mathrm{~cm}$ de profundidade (Tabela 4). Para Vasconcelos et al. (2014) o aumento do grau de compactação nas áreas com aplicação de vinhaça proporcionaram redução de, aproximadamente, 68,70 e $78 \%$ da Pt, respectivamente, nas profundidades de $0-20,20$ 40 e 40-60 cm em relação à MN.

Avaliando o manejo intensivo proporcionado às áreas canavieiras, Garbiatti et al. (2011) constataram redução da Pt nas profundidades de 5 a $20 \mathrm{~cm}$ no sistema de colheita manual de cana queimada, aumento na área de colheita manual e resultados intermediários no sistema de colheita mecanizada, enquanto Zolin et al. (2011) confirmaram redução da Pt na camada de 0-15 $\mathrm{cm}$. Os estudos de Streck et al. (2004) em Argissolo Vermelho-Amarelo indicaram que o tráfego de máquinas tornou-se um dos fatores responsáveis para a diminuição da Pt.

As mudanças na $\mathrm{Pt}$ nas áreas agrícolas diminuiu significativamente a $\theta$ s em comparação com a MN (Tabela 6). Quando se analisa a influência da profundidade de solo no armazenamento de água, constata-se a que a umidade foi reduzida a partir de $20 \mathrm{~cm}$ em, praticamente, todos os manejos.

Tabela 6. Valores de $\theta \mathrm{s}, \mathrm{cm}^{3} \mathrm{~cm}^{-3}$, considerando a interação entre os sistemas de manejo e as profundidades avaliados

\begin{tabular}{|c|c|c|c|c|c|c|c|c|c|c|}
\hline \multirow{3}{*}{$\frac{\text { Manejos }}{\text { SV SQ }}$} & \multicolumn{10}{|c|}{ Profundidade $(\mathrm{cm})$} \\
\hline & \multicolumn{2}{|c|}{$0-10$} & \multicolumn{2}{|c|}{$10-20$} & \multicolumn{2}{|c|}{$20-30$} & \multicolumn{2}{|c|}{$30-40$} & \multicolumn{2}{|c|}{$40-50$} \\
\hline & 0,31 & $c A$ & 0,20 & $b B$ & 0,27 & $b B$ & 0,27 & $b c B$ & 0,29 & $C A B$ \\
\hline SV CQ & 0,34 & bcA & 0,29 & $b c B$ & 0,27 & bB & 0,26 & $c B$ & 0,29 & $C B$ \\
\hline CV CQ & 0,31 & $c A$ & 0,29 & $b c A$ & 0,29 & bA & 0,29 & bcA & 0,30 & bcA \\
\hline CV SQ & 0,35 & bA & 0,31 & $b B C$ & 0,29 & bC & 0,30 & $b B C$ & 0,32 & bAB \\
\hline $\mathrm{MN}$ & 0,44 & $\mathrm{aA}$ & 0,41 & $a A B$ & 0,38 & $a B C$ & 0,37 & $\mathrm{aC}$ & 0,36 & $\mathrm{aC}$ \\
\hline
\end{tabular}

$\mathrm{CV}(\%)$ geral $=7,5$; DMS para colunas $=0,033$ (letras minúsculas); DMS para linhas $=0,033$ (letras maiúsculas). Letras iguais, minúsculas na coluna e maiúsculas na linha, não diferem entre si a $5 \%$ de probabilidade pelo teste Tukey. 
O efeito da mecanização nas propriedades físicas do solo deve ser avaliado com atenção, pois o tráfego de máquinas intenso também pode gerar consequências negativas à porosidade, prejudicando a infiltração e diminuindo a capacidade de retenção de água do solo (CEDDIA et al., 1999). As alterações na distribuição dos diâmetros dos poros, em decorrência dos manejos efetuados nas áreas canavieiras, podem se relacionar com a capacidade de retenção de umidade do solo (SILVA et al., 2005). Neste caso, também é possível realizar a associação da $\theta$ s com teor de MOS (Tabela 3), diante de sua capacidade de aumentar a adsorção de água do solo (CAMARGO; ALLEONI, 1997).

\section{CONCLUSÕES}

O teor de matéria orgânica e a densidade do solo não foram influenciados pelos manejos estudados.

A densidade do solo foi significativamente menor na camada de $0-10 \mathrm{~cm}$, independente do manejo.

A porosidade total e a umidade de saturação do solo foram reduzidas a partir de 20 $\mathrm{cm}$ de profundidade, independente do manejo.

A mudança no uso do solo da condição de área nativa para agrícola influenciou negativamente todos os parâmetros avaliados.

A avaliação da fertilidade e da física de solo devem ser contempladas em estudos de longa duração.

\section{AGRADECIMENTOS}

Os autores agradecem à equipe técnica da empresa do setor de bioenergia, pela cessão das áreas utilizadas para a coleta dos dados e pelo apoio logístico. Um agradecimento especial é destinado à Universidade do Oeste Paulista, pelo apoio financeiro e de infraestrutura para a realização das análises físicas.

\section{REFERÊNCIAS}

ABIVEN, S.; MENASSERI, S.; CHENU, C. The effects of organic inputs over time on soil aggregate stability - A literature analysis. Soil Biology and Biochemistry, v. 41, n.1, p. 1-12, 2009. https://doi.org/10.1016/j.soilbio.2008.09.015

ANDRIOLI, I. Efeitos da vinhaça em algumas propriedades químicas e físicas de um Latossolo Vermelho-Escuro textura média. 1986. 85 f. Tese (Doutorado) - Escola Superior de Agricultura "Luiz de Queiroz" Piracicaba, Piracicaba-SP, 1986.
BAYER, C.; MIELNICZUK, J.; MARTIN-NETO, L. Efeito de sistemas de preparo de cultura na dinâmica da matéria orgânica e na mitigação das emissões de $\mathrm{CO}_{2}$. Revista Brasileira de Ciência do Solo, v. 24, n.3, p. 599-607, 2000 https://doi.org/10.1590/S0100-

06832000000300013

BRAIDA, J.A.; REICHERT, J.M.; VEIGA, M.; REINERT, D.J. Resíduos vegetais na superfície e carbono orgânico do solo e suas relações com a densidade máxima obtida no ensaio Proctor. Revista Brasileira de Ciência do Solo, v. 30, n. 4, p. 605-614, 2006. https://doi.org/10.1590/S010006832006000400001

BRONICK, C.J.; LAL, R. Soil structure and management: a review. Geoderma, v. 124, n. 1-2, p. 3-22, 2005.

https://doi.org/10.1016/i.geoderma.2004.03.005

CAMARGO, O.A.; ALLEONI, L.R.F. Compactação do solo e o desenvolvimento das plantas. Piracicaba: Escola Superior de Agricultura Luiz de Queiroz, 1997.

CANELLAS, L.P., VELLOSO, A.C.X., MARCIANO, C.R., RAMALHO, J.F.G.P., RUMJANEK, V.M., REZENDE, C.E.; SANTOS, G.A. Propriedades químicas de um Cambissolo cultivado com canade-açúcar, com preservação do palhiço e adição de vinhaça por longo tempo. Revista Brasileira de Ciência do Solo, v. 27, n. 5, p. 935-944, 2003. https://doi.org/10.1590/S0100$\underline{06832003000500018}$

CARDIN, C.A.; SANTOS, C. H.; ESCARMÍNIO, M.A. Impacts of vinasse and methods of sugarcane harvesting on the availability of $K$ and carbon stock of an Argisol. Revista Ceres, v. 63, n. 1, p. 95-102, 2016. https://doi.org/10.1590/0034737X201663010013

CEDDIA, M.B.; ANJOS, L.H.C.; LIMA, E.; RAVELLI NETO, A.; SILVA, L.A. Sistemas de colheita da cana-de-açúcar e alterações nas propriedades físicas de um solo Podzólico Amarelo no Estado do Espírito Santo. Pesquisa Agropecuária Brasileira, p. 34, n. 8, p. 1467-1473, 1999. https://doi.org/10.1590/S0100$\underline{204 X 1999000800019}$ 
CONAB. Companhia Nacional de Abastecimento. Acompanhamento de safra brasileira: cana-deaçúcar. Quarto levantamento: safra 2017/2018. 2017. Disponível em: <http://www.conab.gov.br>. Acessado em: 11 fev. 2018.

EMBRAPA. Centro Nacional de Pesquisa de Solos. Manual de métodos de análises de solo. Rio de Janeiro: Centro Nacional de Pesquisa de Solos, 1997.

FREDDI, O.S.; CENTURION, J.F.; BEUTLER, A.N.; ARATANI, R.G.; LEONEL, C.L. Compactação do solo no crescimento radicular e produtividade da cultura do milho. Revista Brasileira de Ciência do Solo, v. 31, n. 4, p. 627-636, 2007. https://doi.org/10.1590/S0100-

06832007000400003

GARBIATE, M. V.; VITORINO, A. C. T.; TOMASINI, B. A., BERGAMIN, A. C.; PANACHUKI, E. Erosão em entre sulcos em área cultivada com cana crua e queimada sob colheita manual e mecanizada. Revista Brasileira de Ciência do Solo, v. 35, n. 6, $\mathrm{p}$ 2145-2155, 2011. https://doi.org/10.1590/S010006832011000600029

IEA. Mecanização da colheita da cana de açúcar atinge $90 \%$ na safra 2016/17. Análise e Indicadores do Agronegócio, v. 12, n. 6, p. 2-6, 2017.

LIMA, A.C.R.; BRUSSAARD, L.; TOTOLA, M.R.; HOOGMOED, W.B.; GOEDE, R.G.M. A functional evaluation of three indicator sets for assessing soil quality. Applied Soil Ecology, v. 64, p. 194200, 2013. https://doi.org/10.1016/j.apsoil.2012.12.009

LUCA, E.F., FELLER C., CERRI C.C., BARTHÉS B., CHAPLOT V., CAMPOS D.C.; MANECHINI C. Avaliação de atributos físicos e estoques de carbono e nitrogênio em solos com queima e sem queima de canavial. Revista Brasileira de Ciência do Solo, v. 32, n. 2, p. 789-800, 2008. https://doi.org/10.1590/s0100-

$\underline{06832008000200033}$

OLIVEIRA, J.C.M.; VAZ, C.M.P.; REICHARDT, K. Efeito do cultivo contínuo da cana-de-açúcar em propriedades físicas de um Latossolo VermelhoEscuro. Scientia Agricola, n. 52, n. 1, p. 50-55,
1995. https://doi.org/10.1590/S010390161995000100009.

OLIVEIRA, V.S.; ROLIM, M.M.; VASCONCELOS, R.F.B.; COSTA, Y.D.J.; PEDROSA, E.M.R. Compactação de um Argissolo Amarelo Distrocoeso submetido a diferentes manejos. Revista Brasileira de Engenharia Agrícola e Ambiental, v.14, n. 9, p.914-920, 2010. https://doi.org/10.1590/S141543662010000900002

OLIVEIRA, V.S.; ROLIM, M.M.; COSTA, Y.D.J.; PEDROSA, E.M.R.; SILVA, E.F.F. Compressibilidade de um Argissolo amarelo distrocoeso submetido $s$ diferentes manejos. Revista Brasileira de Engenharia Agrícola e Ambiental, v. 15, n. 5, p. 435-442, 2011. https://doi.org/10.1590/S141543662011000500001

OLIVEIRA, A.P.P.; LIMA, E.; ANJOS, L.H.C.; ZONTA, E.; PEREIRA, M.G. Sistemas de colheita da canade-açúcar: Conhecimento atual sobre modificações em atributos de solos de tabuleiro. Revista Brasileira de Engenharia Agrícola e Ambiental, v. 18, n. 9, p. 939-947, 2014. DOI: https://doi.org/10.1590/18071929/agriambi.v18n09p939-947

RAIJ, B.V.; ANDRADE, J.C.; CANTARELLA, H.; QUAGGIO, J.A. Análise química para avaliação da fertilidade de solos tropicais. Campinas: Instituto Agronômico, 2001.

SILVA, A.J.N.; CABEDA, M.S.V.; LIMA, J.F.W.F. Efeito de sistemas de uso e manejo nas propriedades físico-hídricas de um Argissolo amarelo de tabuleiro costeiro. Revista Brasileira de Ciência do Solo, v. 29, n. 6, p. 833-842, 2005. https://doi.org/10.1590/S010006832005000600001

SOUZA Z.M.; PRADO R.M.; PAIXÃO A.C.S.; CESARIN, L.G. Sistemas de colheita e manejo da palhada de cana-de-açúcar. Pesquisa Agropecuária Brasileira, n. 40, n. 3, p. 271-278, $2005 . \quad$ https://doi.org/10.1590/s0100204X2005000300011

SOUZA, H. A., MARCELO, A. V.; CENTURION, J. F. Carbono orgânico e agregação de um Latossolo Vermelho com colheita mecanizada de cana-deaçúcar. Revista Ciência Agronômica, Fortaleza, v. 43, n.4, p.658-663, 2012. 
http://dx.doi.org/10.1590/S1806-

$\underline{66902012000400006}$

STRECK, C.A.; REINERT, D.J.; REICHERT, J.M.; KAISER, D.R. Modificações em propriedades físicas com a compactação do solo causada pelo tráfego induzido de um trator em plantio direto. Ciência Rural, v. 34, n. 3, 755-760, 2004. http://dx.doi.org/10.1590/S0103-

$\underline{84782004000300016}$

VASCONCELOS, R.F.B.; SOUZA, E.R.; CANTALICE, J.R.B.; SILVA, L.S. Qualidade física de Latossolo Amarelo de tabuleiros costeiros em diferentes sistemas de manejo da cana-de-açúcar. Revista Brasileira de Engenharia Agrícola e Ambiental, v.18, n.4, p.381-386, 2014. https://doi.org/10.1590/S1415-

$\underline{43662010000900002}$

VIANA, E.T.; BATISTA, M.A.; TORMENA, C.A.; COSTA, C.S. da; INOUE, T.T. Atributos físicos e carbono orgânico em latossolo vermelho sob diferentes sistemas de uso e manejo. Revista Brasileira de Ciência do Solo, v. 35, n. 6, p. 21052114, 2011. http://dx.doi.org/10.1590/S0100$\underline{06832011000600025}$

ZOLIN, C.A.; PAULINO J.; BERTONHA A., FREITAS P.S.L.; FOLEGATTI, M.V. Estudo exploratório do uso da vinhaça ao longo do tempo. I. Características do solo. Revista Brasileira de Engenharia Agrícola Ambiental, v. 15, n. 1, p. 2228, 2011. http://dx.doi.org/10.1590/S1415$\underline{43662011000100004}$

Recebido para publicação em 15/12/2017

Revisado em21/02/2018

Aceito em 26/01/2018 